\title{
Spacetime encodings. I. A spacetime reconstruction problem
}

\author{
Jeandrew Brink \\ Theoretical Astrophysics, California Institute of Technology, Pasadena, California 91103, USA \\ (Received 8 July 2008; published 5 November 2008)
}

\begin{abstract}
This paper explores features of an idealized mathematical machine (algorithm) that would be capable of reconstructing the gravitational nature (the multipolar structure or spacetime metric) of a compact object, by observing gravitational radiation emitted by a small object that orbits and spirals into it. An outline is given of the mathematical developments that must be carried out in order to construct such a machine.
\end{abstract}

DOI: 10.1103/PhysRevD.78.102001

PACS numbers: 04.80.Cc, 04.20.Jb, 04.30.Db

\section{INTRODUCTION}

Gravitational wave detectors such as LIGO (Laser Interferometer Gravitational Wave Observatory) are rapidly increasing their sensitivity, making precise measurement of waveforms emanating from massive gravitating objects a reality in the near future. The launch of LISA (Laser Interferometer Space Antenna) will further increase our observational capacity [1].

One method of mapping out the spacetime of strong field regions is observing the waveforms of extreme and intermediate mass ratio inspirals (EMRIs and IMRIs). The physical scenario is presented in Fig. 1: A low-mass inspiraling object (a "probe", e.g., a particle or carrot or neutron star) moves through the background spacetime on nearly geodesic orbits around a central compact object. As it does so it samples the geodesic structure of the background manifold, as warped by the compact object, and broadcasts this information via gravitational radiation to detectors such as LIGO. The intrinsic periods associated with the particle motion, and the change of these periods as the particle inspirals give us a way of characterizing the spacetime in which the particle is moving.

The current mathematical formulation of this problem, waveform generation and data analysis techniques, is sufficient only for "observing" Kerr EMRIs [2-4]. In effect, current techniques presuppose that the central object is a Kerr black hole, i.e. the unique Kerr spacetime favored by the no-hair theorems [5,6]. In other words, it is assumed that the axioms of cosmic censorship and causality hold and that the only parameters to be determined are the mass and spin of the black hole and orbital parameters of the probe.

Suppose, however, one would like to entertain the idea that something more exotic [7-9] may be created in the strong field regions of the Universe, and would like to find a way of observing, rather than presupposing, what these features are. Suppose that one would like to put ideas such as cosmic censorship and causality to an experimental test rather than using them to aid the data analysis. How would one go about, in effect, drawing the bunny out of the hat in Fig. 1, by watching the radiation emitted by the inspiraling object?
The outline of a mathematical machine, although complex, that could possibly do so, is the subject of this paper. Subsequent papers in this series, [10-12], will develop tools that may make possible such a machine.

In Sec. II, we comment on the existing concept for EMRI searches in gravitational wave (GW) detector data and highlight at each step the mathematical features that make it possible.

In Sec. III, we give a suggested formulation of the problem that could, in principle, encompass, as the central object, all stationary axisymmetric vacuum spacetimes, and we highlight ideas from integrable systems and exact solutions of the Einstein equations that could underpin the desired machine.

Finally, in Sec. III, we also identify crucial points in the mathematical understanding of the field equations that must be sorted out in order to make such a machine viable.

\section{THE EXISTING EMRI WAVEFORM GENERATION MACHINE}

Current calculations of EMRI waveforms have been restricted mainly to inspirals around Kerr black holes. A schematic sketch of the current waveform generation technique, as implemented by Drasco and Hughes [4], and a search algorithm are given in Fig. 2.

In step A, it is assumed that the probe particle is moving around a Kerr black hole whose mass and spin are known. The motion of the probe particle results in a perturbation

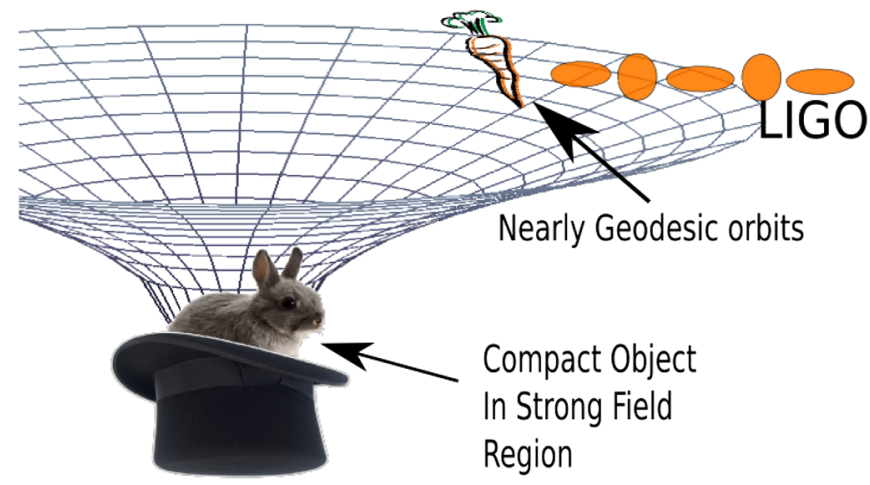

FIG. 1 (color online). Caricature of an EMRI. 


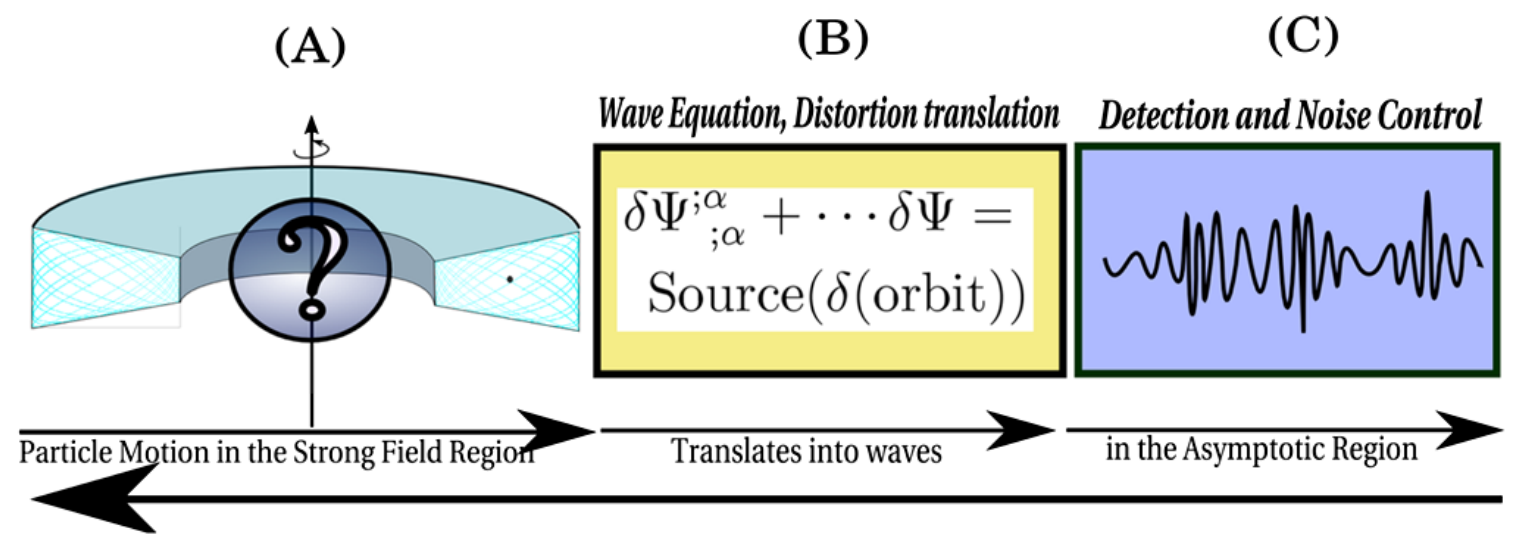

FIG. 2 (color online). EMRI waveform generation machine.

on the background spacetime which is translated using Teukolsky's equation $[4,13]$ into a waveform in the asymptotic region where the detectors are located, step B. The observational step $\mathrm{C}$ involves detection and estimation of the source's parameters via matched filtering. The noisy nature of the data of all $\mathrm{GW}$ detectors makes this final step a necessity: a method for differentiating between features of the detected signal that have their origin in the EMRI signal and those that have their origin in detector noise is required. Once a detection is made and the parameter estimation step for the mass, spin, and orbital parameters of the inspiraling object is conducted, we attribute the gravitational wave event to having been triggered by an inspiral around a Kerr black hole. The uniqueness of this identification is in most cases assumed.

Let us now analyze each step of the process in greater detail and note the features of the calculation that make it tractable. The Kerr spacetime is of Petrov type D (for the definition of Petrov type see [14]) and admits a full set of isolating integrals, or constants of motion; namely, the rest mass $\mu$ of the particle, energy $E$, axial angular momentum $L_{z}$, and the Carter constant $Q$. While the first three constants can be trivially obtained from the metric symmetries, Eq. (1), the Carter constant $Q$ is more subtle. Discovered by separation of the Hamilton Jacobi equation by Carter in 1968 [15], it plays an important role in every step of the calculation. In step A, the constants of motion (or action variables, as they are known in the field of dynamical systems) give us the power to fully describe the orbit: the action variables uniquely identify the orbit of the test particle around the compact object and describe its physical confinement. The angle variables identify where on the trajectory the particle is located at a given time, while traversing the orbit.

In step B, the particle's motion in its orbit serves as a source of the Teukolsky equation, which is used to translate the perturbations caused by the particle in the strong field region into the gravitational waves we observe with our detectors. The Teukolsky equation can be solved by means of separation of variables [13]; the analysis performed by
Teukolsky is only valid in Petrov type D spacetimes. It is this separability feature of the Teukolsky equation that is exploited by Drasco and Hughes [4] to perform the translation of particle motion from the strong field region into gravitational waves in the asymptotic region. There turns out to be a very deep relationship between the separability of differential operators such as those governing the Teukolsky equation, and the existence of a second order Killing tensor on Petrov type D spacetimes (see Ref. [16], chapter 5 and the references therein). In the Kerr spacetime, the Carter constant can be attributed to the existence of a second order Killing tensor, and the Teukolsky equation's separability in Boyer-Lindquist coordinates can be seen as a natural result.

In step $\mathrm{C}$, the waveforms computed for Kerr in steps $\mathrm{A}$ and $\mathrm{B}$ are used as templates for matched filtering. The effect of the Carter constant is evident in these templates, in that the Fourier spectra of the waveforms themselves are built up of harmonics of three fundamental frequencies that characterize the orbit (the three frequencies map directly onto $E, L_{z}, Q$ ). The observation that the existence of four isolating integrals implies that any observable related to bound geodesic orbital motion can be decomposed into a Fourier series characterized by three discrete frequencies was first made by Wolfram Schmidt [17]. This observation underpins the mode decomposition numerically implemented by Drasco and Hughes [4] as well as observational signature of the waveform.

The matched-filtering step is essential in noisy experimental environments. However, matched filtering is also very limiting, in that it requires that we know the form of the templates before performing data analysis. As a result, the current EMRI template bank consists mainly of waveforms generated in Kerr spacetimes.

The EMRI waveform generation machine presented in the discussion thus far will produce waveforms that accurately model radiation from an orbit of an idealized object of infinitesimal mass around a central Kerr black hole. The moment one departs from this idealization, and introduces the effects of a finite mass ratio [18-23], the spin of the 
orbiting particle [24,25], the interaction of particle with the matter in the background spacetime [26-28] or the modification of the background spacetime itself by means of deviations in structure of the central object from Kerr $[29,30]$, the frequencies begin to drift with respect to the expected geodesic Kerr values. Each effect just mentioned introduces one or more small parameters that characterize the deviation. Each parameter in turn introduces a proliferation of templates and possible waveforms with different scalings and time scales for the competing small effects. The first two effects can be modeled as an effective selfforce which pushes the probing particle off geodesics and thus modifies the frequencies during an inspiral. The selfforce has been extensively treated in the literature cited above. While it is not the focus of this paper it should be noted that in order to ultimately make spacetime reconstruction practical the orbital modifications due to these forces need to be quantified very precisely and incorporated in the final analysis, a point that will be further addressed in Sec. IIIC. The effect of the modification of the background spacetime on the geodesic structure is less well understood and is the focus of this and subsequent papers in the series [10-12]. Unfortunately, nature is unlikely to broadcast a priori which small effect is causing the orbit to deviate from a Kerr geodesic. A practical method for reconstructing spacetime will need to be able to discern which small effect is being observed in the waveform before attributing a definitive cause to a particular measurement event.

Isolated examples of explorations of spacetime mapping or the reconstruction of the multipole-moment structure of the central object from observed gravitational radiation, include Ryan's exploration into the feasibility of detection of the multipole moments of a boson star [8]. Collins and Hughes [29] provide an important contribution toward the formalism for mapping spacetime around "bumpy black holes" by studying the orbits confined to the equatorial plane around "bumpy" static objects described by the Weyl class of solutions to the vacuum Einstein equations. Glampedakis and Babak [30] explored the possibility of detecting waveforms generated by an EMRI in a quasiKerr spacetime, represented by a Hartle-Thorne metric which captures effects corresponding to quadrupole deviations from Kerr. The idea of a confusion problem, alluded to in the previous paragraph, where the waveform emanating from a non-Kerr event can be mimicked by a Kerr EMRI with different orbital parameters or vise versa is aptly demonstrated in [30]. In should be noted that as additional small effects as outlined in the previous paragraph are taken into consideration the state of confusion is likely to increase.

The central philosophy advocated by $[29,30]$ is that there is an enormous amount of astrophysical and theoretical evidence in favor that "black hole candidates" are "black holes" as described in general relativity. They suggest treating the problem as a null experiment, in which the null hypothesis is that those objects are just Kerr black holes. A number of relevant alternatives are introduced as "straw men" for which templates are generated. The null hypothesis is then tested by limiting the "bumps" [29] allowed or the deviation of the Kerr quadrupole [30] by using matched filtering and parameter estimation. The number of parameters introduced are limited by the choice of straw men, but so too is the power of the result. If the hypothesis holds, one can only claim, upon completion of a test, that Kerr produces a better model of the waveform than any of the postulated straw men. If the null hypothesis fails and a particular straw man produces a better match, the interpretation may be ambiguous. One of the more frightening scenarios within the paradigm of the null hypothesis test is that one of the other small effects previously mentioned consistent with the Kerr model has a larger overlap with a deceptive straw man waveform than the possibly incomplete templates taken to represent Kerr. In this event the Kerr null hypothesis will be invalidated simply because the waveform resulting from a Kerr inspiral was not well enough modeled or known. One thus has to ensure that a decisive straw man does not correlate with any other possible physical effect consistent with the Kerr hypothesis. The most obvious effect that has to be accounted for being that of the self-force. The null hypothesis scenario sketched in $[29,30]$ within the context of straw man models containing a few parameters serves well to elucidate differences in waveform due to a spacetime with or without a certain feature depending on the analysis and the difficulties faced in resolving this binary choice. The observed confusion problem, however, gives a very stern warning against a case of mistaken identity when using this approach. An experimentally decisive straw man model need necessarily bear knowledge of all other physical effects consistent with the Kerr inspiral and consequently have a large number of parameters to be determined by means of matched filtering. Furthermore the point during the modeling process of a Kerr event at which one begins to deem it appropriate for a deceptive straw man to change character and become a decisive straw man must be established.

The matched-filtering approach hampers observation by requiring that one initially compute the model of the central object, work out possible inspiral templates from the resulting spacetime, and then conduct the matching and parameter estimation. An effective spacetime mapping algorithm, beyond Kerr spacetimes, would require an enormous number of templates and even then one could not possibly hope to cover all possible scenarios. In effect, the observational power of the LIGO and LISA detectors in the EMRI inspiral problem is limited by the models we can conceive and calculate. To date, no general framework exists that will allow us to effectively map the strong field region around an unknown object. 
The approach followed in this paper adheres to a philosophy employed with great success in the past in the field of dynamical systems and chaos, namely, that on occasion the general problem is easier to solve than a special case. It strives to go after the general structure of the field equations in the restricted context of stationary axisymmetric vacuum (SAV) spacetimes, rather than explicitly referring to a particular spacetime metric. While the reader may be dubious about the practicality of such an approach, it is thought that addressing the problem within this broader context and identifying initially what is desired, necessary, possible, and ultimately even what is possibly unmeasurable, may increase the scope of discussion and final practical realization. Ultimately, however, well-informed and well-chosen special cases are invaluable in furthering our understanding, and a practical solution will be the product of both approaches.

In the next section, we formulate the EMRI problem for general SAV spacetimes, and we suggest methods in which the ideas of integrable systems can be applied to make a general detection algorithm possible.

\section{FORMULATION OF THE EMRI PROBLEM FOR AXISYMMETRIC STATIONARY SPACETIMES}

Consider a central body with arbitrary multipole moments and a probe particle moving in the vacuum spacetime around it. The line element of this spacetime can be represented in the Lewis Papapetrou form

$$
\begin{aligned}
d s^{2}= & k^{2} e^{-2 \psi}\left[e^{2 \gamma}\left(d \rho^{2}+d z^{2}\right)+R^{2} d \phi^{2}\right] \\
& -e^{2 \psi}(d t-\omega d \phi)^{2},
\end{aligned}
$$

where $\psi, \gamma, \omega$, and $R$ are functions of $\rho$ and $z$, and $k$ is a real constant. The vacuum field equations relate these functions to solutions of the Ernst equation for the complex potential $\mathcal{E}$,

$$
\Re(\mathcal{E}) \bar{\nabla}^{2} \mathcal{E}=\bar{\nabla} \mathcal{E} \cdot \bar{\nabla} \mathcal{E},
$$

where $\bar{\nabla}^{2}=\partial_{\rho \rho}+\frac{1}{\rho} \partial_{\rho}+\partial_{z z}, \bar{\nabla}=\left(\partial_{\rho}, \partial_{z}\right)$, and the dot is the flat-space inner product. In particular, the function $e^{2 \psi}=\Re(\mathcal{E})$ denotes the real part of the potential. The functions $\gamma$ and $\omega$ can be obtained by means of line integrals of the Ernst potential once it is known. The harmonic function $R$ obeys the equation $R_{z z}+R_{\rho \rho}=0$ and represents a gauge freedom still present in the metric. The choice $R=\rho$ is often made.

A particular solution of the SAV field equations can be identified by means of a bi-infinite sequence of numbers physically interpreted as multipole moments $M_{i}$ and $S_{i}$. This corresponds to choosing a particular element of the Geroch group. As first conjectured by Geroch $[31,32]$ and later proved by Hoenselaers, Kinnersley, Xanthopoulos
(HKX), and Chitre [33-37] in the late 1970s in a series of papers that lead to the HKX transformations, these numbers uniquely identify a spacetime. Once they are known, the spacetime is in principle determined. Subsequently, a number of other solution-generating techniques have been developed that allow one to determine the explicit form of the functions in the metric (1) by mapping a given solution of (2) onto another [38].

We speculate that it may be possible to exploit these mappings to help develop an algorithm that could in principle limit, if not completely determine, the multipole structure of a spacetime from its EMRI inspiral waves. We further speculate that it may be possible to do so without resorting to matched filtering and its need for $a$ priori guessing the structure of the central object.

There are several uncertainties implicit in expanding the model shown in Fig. 2 from Kerr to a general method for mapping spacetime, all of which have to be addressed before a spacetime reconstruction algorithm becomes practical and before we can determine how much information can, in practice, be gleaned from an EMRI inspiral event. These uncertainties include (A) whether or not an explicit action-angle variable prescription can be found that gives us access to the full description of the probing particle's geodesic orbit. (B) If (A) is indeed possible in large regions of more general spacetimes, is it feasible to attempt to explore the perturbation problem on a general background and what form would that calculation take? (C) Observationally, one is only privy to partial knowledge of the gravitational wave emission originating from a sequence of geodesic "snapshot" orbits (portion of the orbit in which the radiation-reaction-induced evolution of integrals of the motion is negligible), as in Fig. 2. The noise of the detector, the effect of the mass of the probing particle on its motion through the background spacetime that moves it off the geodesic trajectory (self-force), the length of the observation or validity of the adiabatic approximation and a zoo of other small effects mentioned in Sec. II, all conspire to complicate the signal. What is sought is a method to extract the signal from the noise and a subsequent representation of that signal that allows one to clarify the nature of the nongeodesic effects and quantify which parameters describing the central object can be obtained with certainty. All of these questions and uncertainties are addressed in the next three paragraphs.

\section{A. Orbital description}

In many calculations in general relativity it is implicitly assumed that it is possible to find four constants of geodesic motion that describe the orbital motion of a test particle, and it is the bias of the author that it is indeed so. However, in the past, such intuition has proven faulty. Poincare's study of the three-body problem and the advent of our understanding of deterministic chaos forever banished the ideal of finding a beautifully simple closed form descrip- 
tion of particle motion in Newtonian gravity [39]. The Hénon-Heiles problem warns that in the event of the system being chaotic, perturbation theory, while it will yield a computational result, will fail to accurately represent the dynamics of the system [40]. Numerical studies into the orbital nature of SAV spacetimes are conducted in [41] and are discussed further in paper II of this series [10]. Understanding the interrelationship between the existence of Killing tensors and the integrability properties of the Ernst equation and solution generation techniques may provide a constructive method of finding the invariants in question; see papers III and IV in this series [11,12]. Such an investigation may also shed light on a possible approach to the resolution of problem (B).

I conclude this subsection with an analogy that strives to illustrate the observational and computational implications in the event that a complete orbital description in terms of four constants of geodesic motion does not exist. The chosen analogy is in terms of fluid flow. Suppose, first, that as in the case of the Kerr spacetime, a complete orbital description exists. In this case, the geodesics are nicely ordered, akin to the streamlines of steady state laminar flow. These lines can be observed by adding a small particle of colored dye to the river and observing its motion as it propagates along the streamlines which are determined by the shape of the river and obstacles in its course. The analog of the self-force can be introduced by adding a weak diffusion gradient across the river. The dye still propagates downstream roughly tracing out the flow as it slowly progresses from streamline to streamline on time scales set by the diffusion gradient. Observing the dye and armed with knowledge of the diffusion process, one can learn about the structure of the river. Suppose now that we have the other extreme, namely, that the geodesic equations are strongly chaotic and no constants of motion can be found. The absence of constants of motion implies that individual orbits defy concise mathematical description; the best analogue for this chaotic state is turbulent flow. This state represents a writhing, churning, and twisting of space and introduces a new very rapid time scale into the problem in the form of the Lyapunov exponent. Careful observation of the haphazard, unpredictable, and unrepeatable trajectory of a single dye particle in turbulent flow will not leave the observer with an accurate impression of the shape of the river or the obstacles in it. In order to glean such information in the event of turbulent flow necessitates a statistical treatment of observations of several particles of dye. As illustrated in [41], the signal originating from a chaotic spacetime will be devoid of periodicity and represent nothing more than noise with certain statistics. It is unclear how in LIGO and LISA observations one would separate this signal from detector noise and thus observe it. If one adds a diffusion gradient to the turbulent river, not much changes. The rapid time scale associated with the turbulent flow will dominate the dynamics and the obser- vational treatment will still by necessity remain statistical, diffusion possibly accounting for a small change in the statistics. What has been presented here are two extreme cases; any good river will demonstrate an array of flow patterns in between. The analogy strives to emphasize the importance of establishing which regime the system is in because it drastically influences the observation techniques employed.

It should be mentioned in closing that the KolmogorovArnold-Moser theorem (KAM) [42] ensures the integrity of the EMRI and self-force programs pursued thus far with respect to the Kerr spacetime. The KAM theorem assures us that systems that are close to integrable systems possessing a full set of constants will also exhibit "laminar like" flow and will not spontaneously erupt into a turbulent froth; the only caveat being that the theorem does not quantify how close.

\section{B. Translation of orbital motion into detection region}

The translation of the effect of particle motion in the strong field region into waves in the asymptotic region and subsequent coupling to the detector requires that one solves the perturbation problem off of all SAV solutions. Although this task may seem daunting, and very little appears to have been done on perturbations off more general SAV backgrounds than Kerr, the idea of GW traveling outward can, in some sense, be viewed as a particle perturbation traveling along a series of plucked strings or geodesics toward the asymptotic region. Just as in the Petrov type D case, the integrability properties of the wave equations (or perturbation equations) in the general spacetimes should be related to the background geodesic structure of the spacetime one is perturbing off. The two aspects of the Teukolsky analysis [13] that make the problem tractable in type D spacetimes, namely, the decoupling of the perturbation equations and separability have subsequently been understood in terms of second order Killing tensors [16]. The extension of this work to higher order Killing tensors would provide a point of entry to solving problem (B).

\section{Detection and noise control}

As one departs from the idealized picture presented in Fig. 2, namely, that of an infinitesimal test mass moving on a geodesic observed for an infinite period of time, broadcasting radiation with distinctive discrete frequencies, a plethora of small effects enter. To date many of the small effects have been explored in isolation. It should, however, be noted that to make spacetime reconstruction practical and to have an unambiguous interpretation of a waveform event within the EMRI paradigm, a careful accounting of these small effects is required. Furthermore, the possibility of one small effect mimicking another or changes in the 
background spacetime presents a very real danger and should be characterized.

The measured signal will have two sources of uncertainty, namely, those due to measurement and model. In addition to the ever present detector noise, errors due to measurement include the band width of the detector, uncertainty as to which part of the orbit is being observed, finite observation time, measurement resolution, and instrument calibration.

Some of the model based sources of uncertainty are summarized in the following paragraph. The list is by no means complete. It serves more to underscore the bewildering complexity of the problem and highlight the need for a global treatment than to provide a resolution of what that treatment should be. The dominant effect that will cause the signal to depart from having discrete frequencies of the idealized waveform generation machine Fig. 2 (provided the background spacetime is integrable) is that of finite mass ratio $\mu / M$. This effect, know as the self-force, leads to a frequency shift of magnitude $\delta \Omega / \Omega \sim(\mu / M)$. The self-force [18-23] will have to be computed to high accuracy before spacetime reconstruction becomes feasible. As argued in Sec. II with regards to the deceptive and decisive straw men, the precision with which the selfforce is known ultimately determines the certainty with which the nature of the spacetime can be determined. A method by which the self-force can be incorporated into a spacetime mapping algorithm is suggested later in this section. The effect of mass ratio is only the first and most dominant of several other effects associated with the structure of the small inspiraling body that may need to be accounted for during spacetime reconstruction program. Another source of geodesic deviation will be due to the spin of the particle, which, via the Papapetrou equation, couples to the background spacetime $[24,25]$. Spin introduces an effective self-force and unfortunately another parameter. The effects of dynamical interactions between the probe particle and the central black hole, through tidal coupling induced orbital energy transfer may also become significant $[43,44]$. Finally, there is always the annoying complexity introduced by the presence of matter [26-28]. This effect, however, causes less worry since there are strong astrophysical arguments that suggest that the EMRI/IMRI systems are expected to be clean, namely, devoid of matter besides that encompassed in the central object and the probing particle [45]. In the event that matter is present, it may either be interacting with the EMRI directly inducing an effective drag force which was shown to be negligible [26] or take the form of an object disjoint from the orbital trajectory considered in [27]. It should be noted that the second case is already included in the general framework, proposed in this paper, versed in terms of the Ernst potential, provided that the matter is stationary axisymmetric, has a compact base of support surrounded by vacuum, and the orbit does not crash into the object.
Given this tabulation of small effects, in addition to the parameters required to describe the structure of the central object one wants to map, one is faced with a choice regarding a detection strategy. The first option is to build an implicit model of bewildering complexity parameterizing the waveform with a huge parameter space. Then, produce a vast array of templates and within the current matched-filtering framework, proceed with the mammoth task of parameter estimation which will be hindered by the measurement sources of error as well as the suitability of choice of model. The alternative is to attempt to lift the restriction, imposed by matched filtering, of having to explicitly know the exact formulation of the model $a$ priori.

A possible scenario in which the matched-filtering criterion could be lifted is by finding an experimental realization of the solution method for the Ernst equation employed by $[46,47]$ and initially given in [48]. What is done is to introduce a linear potential matrix $\Phi$ much like a wave function in quantum mechanics. In the equation governing $\Phi$, the Ernst potential enters as an unknown field, a quantum mechanical potential well per analogy. A great deal is known about the properties of $\Phi$ [46], without a priori specifying the explicit gravitational field or Ernst potential involved. In effect, the $\Phi$ serves as a carrier or equivalence class for the gravitational potential being observed allowing its properties to be known without specifying the entity itself.

An example of using the known properties of the solutions of an equation to aid detection within a noisy environment, without explicitly modeling the waveform and thus knowing the source of the waves, can be found in the form of the Korteweg-de Vries (KdV) equation describing shallow water waves [49]. This example is much simpler than the SAV problem and is accompanied by tangible physical interpretation. Furthermore, the KdV equation shares many of the same mathematical properties as the GW, SAV problem, and I speculate that it may be worthwhile exploring to provide insight on how to proceed in the GW case. The KdV equation admits solution by means of the inverse scattering method described in the previous paragraph, albeit much less complicated [46,49]. One of the features of the solution identified in the analysis is the dispersion relation. If one is unfortunate enough to attempt to detect shallow water waves on a pond while one's child is splashing in the foreground of the machine, filtering the data using this dispersion relationship, and some knowledge of the functional form of the waves originating further out may suffice in removing the child from the measurement without ruining a good day's play. How exactly, to effect such a filter for a gravitational wave experiment is at present unclear. Two things, however, are certain: we cannot remove the noisy child and, without the filter, our observational power in the context of spacetime mapping is limited. 
For our SAV gravitational wave problem, an approach to the representation of the GW data could be as follows. Observe that the solution to the geodesics equations of Kerr can be written down in terms of Weierstrass's elliptic functions [50], the poles of which are related to the constants of motion, or Killing tensors. Identify segments of the real waveform with the poles associated with the corresponding snapshot waveform. This will allow us to compute pole tracks as the particle moves from geodesic to geodesic. The self-force calculations should provide the theoretically expected track or more accurately, a family of expected tracks parameterized by the mass ratio. In a general spacetime mapping setting, make a similar identification and suppose, initially, that the inspiral is around a Kerr object. Should the pole track begin to deviate from Kerr, systematically adjust the lower order multipole moments of the model so that the observed track best matches the theoretical one. For this the self-force calculation on a general background would be required.

An expression of the metric that is explicitly parametrized by means of the multipole moments will facilitate calculations. In practice, for static spacetimes which are a member of the Weyl class [51], a metric which is parametrized by means of all the mass multipoles already exists. For SAV spacetimes an explicit form of the metric is not known, but known special cases may be helpful in developing our proposed techniques: The Manko-Novikov spacetime [52] provides an explicit metric in which all mass moments and some, but not all, the spin moments appear explicitly in the metric. Other metrics that would be of astrophysical interest and for which the Ernst potential is explicitly known are those of a compact object surrounded by a disk [46].

\section{CONCLUSION}

There will always remain experimental uncertainty as to how well one can determine the structure of the central object and thus the extent to which one can verify the validity of the no-hair theorems [5] or confirm the existence of more complex central objects. Ironically, quantum mechanics and the act of measurement itself force us to play dice in determining the details of Einstein's theory in practice.

Possibly the experimental and data analysis challenge is to find the means by which we can learn the most. If the relationship between curvature content of the Weyl tensor, as encoded in the Ernst potential, and the geodesic structure, can be understood in detail and appropriately exploited, it may lead to a powerful experimental application of the mathematical development in the field of exact solutions over the last few decades. A possible framework by which this can be done has been suggested in this paper.

This paper is presented as a question about the feasibility of this mathematical machine. You are encouraged to find its flaws. In subsequent papers [10-12], some of the ideas presented here will be placed on a firmer mathematical footing, thus laying the foundation for the construction of this machine.

\section{ACKNOWLEDGMENTS}

I would like to thank Duncan Brown, Ilya Mandel, Kip Thorne and Michele Vallisneri for many useful discussions and good advice. I would like to thank the anonymous referee whose suggestions greatly improved the manuscript.
[1] D. A. Brown, J. Brink, H. Fang, J. R. Gair, C. Li, G. Lovelace, I. Mandel, and K. S. Thorne, Phys. Rev. Lett. 99, 201102 (2007).

[2] P. A. Sundararajan, G. Khanna, and S. A. Hughes, Phys. Rev. D 76, 104005 (2007).

[3] P. A. Sundararajan, G. Khanna, S. A. Hughes, and S. Drasco, Phys. Rev. D 78, 024022 (2008).

[4] S. Drasco and S. A. Hughes, Phys. Rev. D 73, 024027 (2006).

[5] P. O. Mazur, arXiv:hep-th/0101012v1.

[6] W. Isreal, Phys. Rev. 164, 1776 (1967).

[7] S. Dubovsky, P. Tinyakov, and M. Zaldarriaga, J. High Energy Phys. 11 (2007) 083.

[8] F. Ryan, Phys. Rev. D 52, 5707 (1995).

[9] N. A. Collins and S. A. Hughes, Phys. Rev. D 69, 124022 (2004).

[10] J. Brink, following Article, Phys. Rev. D 78, 102002 (2008).
[11] J. Brink, "III. Second order Killing Tensors" (unpublished).

[12] J. Brink, “IV. Relationship between Weyl Curvature and Killing Tensors in SAV Spacetimes" (unpublished).

[13] S. A. Teukolsky, Astrophys. J. 185, 635 (1973).

[14] Solutions of Einstein's Equations: Techniques and Results, Proceedings of the International Seminar on Exact Solutions of Einstein's Equations, edited by C. Hoenselaers and W. Dietz (Springer-Verlag, Berlin, 1983).

[15] B. Carter, Phys. Rev. 174, 1559 (1968).

[16] J. Kress, Ph.D. thesis, University of Newcastle (N. S. W.), 1997, http://web.maths.unsw.edu.au/ jonathan/thesis/.

[17] W. Schmidt, Classical Quantum Gravity 19, 2743 (2002).

[18] A. Pound, E. Poisson, and B. G. Nickel, Phys. Rev. D 72, 124001 (2005).

[19] Y. Mino, Phys. Rev. D 67, 084027 (2003).

[20] Y. Mino, Prog. Theor. Phys. 113, 733 (2005).

[21] T. Hinderer and E. E. Flanagan, Phys. Rev. D 78, 064028 
(2008).

[22] S. Drasco, É.É. Flanagan, and S. A. Hughes, Classical Quantum Gravity 22, S801 (2005).

[23] A. Pound and E. Poisson, Phys. Rev. D 77, 044013 (2008).

[24] L. M. Burko, Phys. Rev. D 69, 044011 (2004).

[25] L. M. Burko, Classical Quantum Gravity 23, 4281 (2006).

[26] R. Narayan, Astrophys. J. 536, 663 (2000).

[27] E. Barausse and L. Rezzolla, Phys. Rev. D 77, 104027 (2008).

[28] E. Barausse, L. Rezzolla, D. Petroff, and M. Ansorg, Phys. Rev. D 75, 064026 (2007).

[29] N. A. Collins and S. A. Hughes, Phys. Rev. D 69, 124022 (2004).

[30] K. Glampedakis and S. Babak, Classical Quantum Gravity 23, 4167 (2006).

[31] R. Geroch, J. Math. Phys. (N.Y.) 12, 918 (1971).

[32] R. Geroch, J. Math. Phys. (N.Y.) 13, 394 (1972).

[33] W. Kinnersley, J. Math. Phys. (N.Y.) 18, 1529 (1977).

[34] W. Kinnersley and D. M. Chitre, J. Math. Phys. (N.Y.) 19, 2037 (1978).

[35] W. Kinnersley and D. M. Chitre, J. Math. Phys. (N.Y.) 18, 1538 (1977).

[36] W. Kinnersley and D. M. Chitre, J. Math. Phys. (N.Y.) 19, 1926 (1978).

[37] C. Hoenselaers, W. Kinnersley, and B. C. Xanthopoulos, J. Math. Phys. (N.Y.) 20, 2530 (1979).

[38] H. Stephani, D. Kramer, M. Maccallum, C. Hoenselaers, and E. Herlt, Exact Solutions of Einstein's Field Equations (Cambridge University Press, Cambridge, England, 2003), 2nd ed..

[39] J.H. Poincaré, Acta Math. 13, 1 (1890).

[40] M. Hénon and C. Heiles, Astron. J. 69, 73 (1964).

[41] J. R. Gair, C. Li, and I. Mandel, Phys. Rev. D 77, 024035 (2008).

[42] M. Tabor, Chaos and Integrability in Nonlinear Dynamics-An Introduction (John Wiley \& Sons, New York, 1989), ISBN 0-471-82728-2.

[43] H. Fang and G. Lovelace, Phys. Rev. D 72, 124016 (2005).

[44] C. Li and G. Lovelace, Phys. Rev. D 77, 064022 (2008).

[45] P. Amaro-Seoane, J. R. Gair, M. Freitag, M. C. Miller, I. Mandel, C.J. Cutler, and S. Babak, Classical Quantum Gravity 24, R113 (2007).

[46] C. Klein and O. Richter, Ernst Equation and Riemann Surfaces (Springer-Verlag, Berlin, Heidelberg, 2005).

[47] C. M. Cosgrove, J. Math. Phys. (N.Y.) 21, 2417 (1980).

[48] G. Neugebauer, J. Phys. A 12, L67 (1979).

[49] P. Drazin and R. Johnson, Solitons: An Introduction (Cambridge University Press, Cambridge, England, 1996).

[50] Bateman Manuscript Project, Higher Transcendental Functions II (McGraw-Hill Book Company, New York, 1953).

[51] H. Weyl, Ann. Phys. (Leipzig) 359, 117 (1917).

[52] V.S. Manko and I.D. Novikov, Classical Quantum Gravity 9, 2477 (1992). 